\title{
Crystal Orientation Angular Resolution with Precession Electron Diffraction
}

\author{
E.F. Rauch ${ }^{1,2}$ and M. Véron ${ }^{1}$ \\ ${ }^{1}$ Laboratoire SIMaP, Grenoble INP - CNRS - UJF, Saint Martin D'Hères Cedex, 38402. France. \\ ${ }^{2}$ CNRS, SIMAP, F-38000 Grenoble, France
}

Nanobeam diffraction mapping is becoming a standard procedure to characterized the structure of material at nanoscale. In this technique, series of diffraction patterns are collected and stored while the focused beam is scanning the sample [1]. Essential properties like the local crystallographic texture or the phase distribution may be mapped through the identification of the successive patterns. Besides, specific post processing algorithms permit structural entities to be highlighted: in particular, standard or combined dark-field images have proved efficient to sort amorphous phases [2] and the newly developed construction of electron diffraction correlation coefficient maps has been shown to retrieve 3D information [3].

For these technics, it is better to collect the diffraction pattern in precessing mode. Indeed, precession electron diffraction (PED) is known to increase the number of reflections and to decrease the dynamic effect on the Bragg reflection intensities, which, in turn, improve the pattern identification [4]. However, it is frequently mentioned that the crystal orientation cannot be retrieved with an angular resolution better than the precession angle. This is because a large fraction of the reciprocal lattice is swept by the Ewald sphere when the beam is rocked and no substantial change is expected in the diffraction pattern if the crystal is tilted by an amount smaller than the precession angle. Although reasonable, this view turns out to be untrue as demonstrated below.

Diffraction patterns were collected continuously for a Si single crystal progressively tilted in a procedure similar to the fast diffraction tomography setup of Gemmi et al [5]. The $1.2^{\circ}$ precession angle was monitored with the Nanomegas Digistar P1000 attachment to a JEOL 2100F TEM. The crystal orientation is computed with the ASTAR system through template matching and compared to the quasi linear evolution expected from the constant angular speed of $0.16 \%$ imposed by the TEM goniometer. The high camera collection speed (100 fps) produces significant noises that was reduced by averaging 50 successive diffraction patterns for each measured point. This leads to a rotation step of $0.08^{\circ}$ per analyzed diffraction pattern. Each of them were compared to a set of pre-calculated templates by image correlation [1]. The orientation step selected to generate the templates was less than $0.05^{\circ}$. The measured deviation from the linear trend is used to weight the angular resolution.

Figure 1.a depicts the progressive misorientation with respect to the initial orientation as deduced from the template matching identification procedure. Although the general trend corresponds to the expected linear one (i.e.: no misindexing), substantial departures from linearity are computed. The standard deviation $\Delta \sigma$ is $0.51^{\circ}$ and consequently the angular resolution $(\sim 2 \Delta \sigma)$ is practically equal to the precession angle. This value is in apparent agreement with the above mentioned limitation. However, the limited accuracy results essentially from the simplified way to generate the templates, the later being computed without considering any modifications of the diffracting signal related to precession. When precession is used, the intensities of the reflections are substantially affected. Fig.2 depicts the evolution of a given Bragg spot along the tilt series. It appears that - as expected - the reflection remains intense for an extended orientation range, that scales with the precession angle. Also, this rocking curve shows that the intensity profile is not constant, but splitted in two peaks separated by twice the precession 
angle. For sake of conciseness, the definite reason that explains this behavior will not be detailed here. For the present purpose it is sufficient to note that this trend is systematically recorded for all Bragg reflections and for all precession angles.

When the templates are computed by including this typical PED profile, they exhibit significant changes with respect to the non-precessed theoretical patterns (Fig.2.b). The improved set of templates being closer from the effective intensity evolution measured for the tilt series, the crystal orientation is determine with an increased accuracy (Fig. 1.b). The standard deviation is dramatically reduced. The angular resolution is of the order of a third of a degree $\left(\Delta \sigma \sim 0.16^{\circ}\right)$, that is nearly four times lower that the precession angle.

[1] E.F. Rauch, M. Véron, Mater. Charact. 98 (2014) 1-9.

[2] E. F. Rauch and M. Véron, Eur. Phys. J. Appl. Phys. (2014) 66: 10701

[3] Á.K. Kiss, E.F. Rauch, J.L. Lábár, Ultramicroscopy 163 (2016) 31-37.

[4] P.A. Midgley, A.S. Eggeman, , IUCrJ 2 (2015) 126-136.

[5] M. Gemmi, M.G.I. LaPlaca, A.S. Galanis, E.F. Rauch, S. Nicolopoulos, J.Appl.Cryst. 48(2015)1-10.
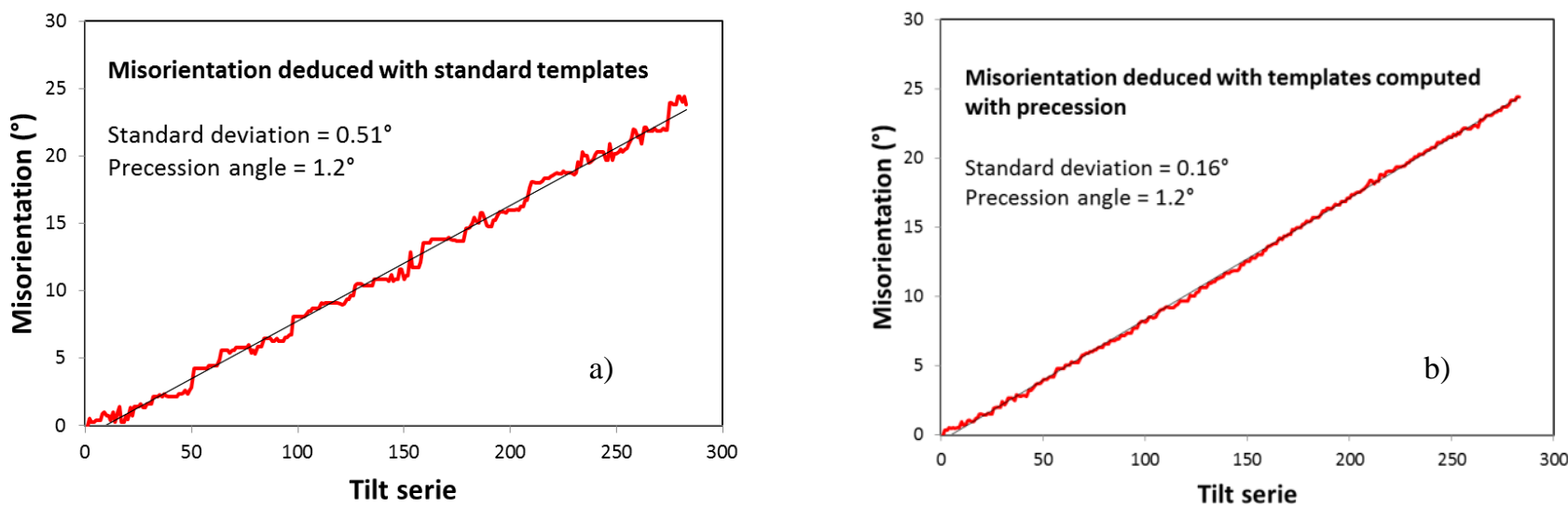

Figure 1. Measured misorientation for a tilted single Si crystal with templates calculated without (a) and with (b) precession.

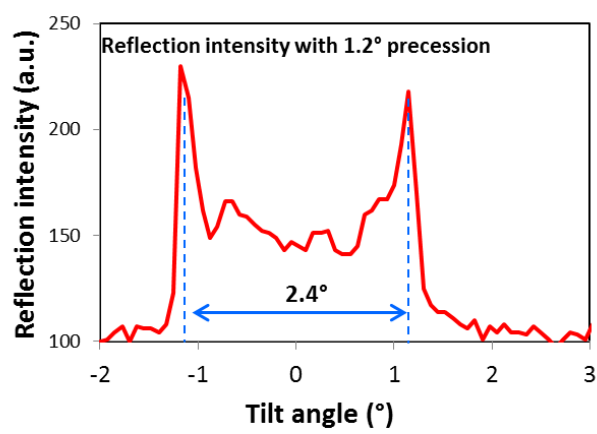

a)

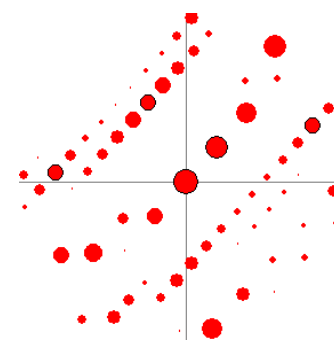

No precession

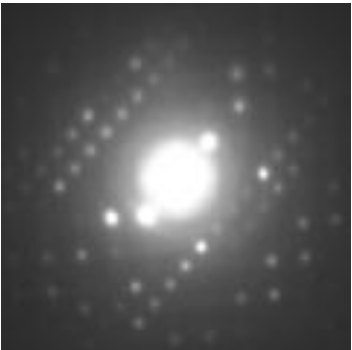

b)

Figure 21. Introducing the Bragg intensity profile pertaining to precession (shown in (a)) in the the template computing leads to theoretical diffraction patterns closer from the measured ones (e.g.: (b)) 